\title{
Horizons/Théâtre
}

Revue d'études théâtrales

\section{Lecture documentarisante et fictionnalisation du réel dans le spectacle Mvet du peuple ékang}

\section{Aristide Sanama Nguillé}

\section{(2) OpenEdition}

1 Journals

Édition électronique

URL : https://journals.openedition.org/ht/966

DOI : $10.4000 /$ ht.966

ISSN : 2678-5420

Éditeur

Presses universitaires de Bordeaux

\section{Édition imprimée}

Date de publication : 31 décembre 2018

Pagination : 36-53

ISBN : 979-10-300-0318-5

ISSN : 2261-4591

\section{Référence électronique}

Aristide Sanama Nguillé, « Lecture documentarisante et fictionnalisation du réel dans le spectacle Mvet du peuple ékang », Horizons/Théâtre [En ligne], 13 | 2018, mis en ligne le 01 janvier 2019, consulté le 17 mai 2022. URL : http://journals.openedition.org/ht/966 ; DOl : https://doi.org/10.4000/ht.966

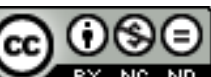

La revue Horizons/Théâtre est mise à disposition selon les termes de la Licence Creative Commons Attribution - Pas d'Utilisation Commerciale - Pas de Modification 4.0 International. 


\section{ARISTIDe SANAMA Nguillé}

Aristide Sanama Nguillé est cinéaste-documentariste, réalisateur et producteur. Son champ d'étude est les Arts du spectacle et cinématographie. Descendant du côté maternel du clan Mvog-Zambo, de la lignée Eba (joueurs de « mvet ») que l'on trouve au Cameroun, au Gabon et en Guinée équatoriale, il est théoricien et praticien de cet instrument qui fonde la cosmogonie du peuple Ekang. Enseignant-assistant à I'Université de Yaoundé I où il est par ailleurs membre du Laboratoire des arts de la scène et de l'écran (LASEC) de la Faculté des Arts, Lettres et Sciences humaines, il présente une thèse de doctorat Ph.D. Formé par I'Institut national de l'audiovisuel de Paris (INA) à la conception et construction des chaînes et multiplex TNT, il est l'auteur de plusieurs documentaires dont La théâtralisation des cérémonies mortuaires en pays Beti (2011), Cameroun, de I'Indépendance à I'Unité nationale (2015), Poterie et métallurgie du bronze : un savoir-faire ancestral en voie de disparition (2017) et Ekang : la complainte du Mvet-oyeng (2017).

\section{Mail: nguillsanama@yahoo.fr}

Résumé : Le nom mvet fait référence à un instrument de musique et à une littérature orale chantée, récitée et accompagnée de cet instrument. Présent au Cameroun, au Gabon, en Guinée équatoriale, au Congo et surl'île de Sao Tomé et Principe, son épopée principale raconte sousforme de fiction, les faits d'armes du peuple Ékang de la forêtéquatoriale. Le spectaculaire du Mvet mêle en effet la réalité à lafiction. Or, il est coutume d'opposer ces deux paradigmes. $C^{\prime}$ est le cas du Mvet, conte qui met en scène des personnages aux pouvoirs surnaturels dans l'univers ékang, un monde bien réel. D'où la question suivante : quelle relation le réel entretient-il avec la fiction dansle récit du Mvet?

Mots-cLés : oralité, Mvet, conte, fiction.

fiction. However, it is customary to oppose these two paradigms. This is the case with Mvet, a tale which stages characters with supernatural powers in the Ekang universe, a very real world. Hence the following question: what relation does reality have with fiction in the Mvet story?

Kerwords : orality, Mvet, tale, fiction. 


\section{Lecture documentarisante et fictionnalisation du réel dans le spectacle Mvet du peuple ékang}

\section{Introduction}

PARLER De LeCTURE DOCUMENTARISANTE dans le spectaculaire du Mvet, c'est rapprocher sa pratique du genre cinématographique qu'est le documentaire. En effet, il existe des similitudes entre le Mvet comme genre épique et le documentaire comme genre cinématographique. Le Mvet est un genre populaire majeur de l'orature des peuples Beti-Bulu-Fang regroupés sous l'appellation Ékang. Il se caractérise par la glorification de ses héros aux pouvoirs surnaturels et des combats entre immortels et mortels voulant conquérir l'éternité (Tsira Ndong Ndoutoume, 1983). Le Mvet en plus d'être un récit est un spectacle vivant où mythes, politique, stratégies militaires et sciences participent à la «mise en scène » pour captiver l'attention du spectateur par l'entremise de la parole du barde «mbomomvet ». Le Mvet est aussi une lecture de la réalité ékang vue sous le prisme du conteur qui l'interprète, lui donne vie (Angèle Christine Ondo, 2014) par ses récits, ses chants et ses silences. Parler du documentaire par ailleurs revient à parler d'un genre filmique qui a caractère de «document » (François Niney, 2000) car il s'appuie sur des faits et des événements vrais pour décrire une certaine réalité ou l'arranger selon les convenances. Pour réaliser un documentaire, il n'est donc pas impossible de faire une «mise en place », c'est-à-dire procéder à des reconstitutions des faits ou éléments manquant pour soutenir le récit ou les silences voulus par le réalisateur. La voix-off présente dans le documentaire sert de trame narrative et vient appuyer en expliquant le récit des événements. On peut donc constater que comme dans le Mvet, la parole occupe aussi une place de choix dans le genre documentaire. Elle est porteuse d'informations mais aussi de significations. Toutefois, en documentant le réel, le Mvet tout comme le documentaire utilise la parole pour raconter l'action ou la faire revivre dans la tête des spectateurs. Les épopées mvet sont accompagnées par des mélodies, des gestuels et des rythmes. La civilisation africaine ayant basé la transmission de son savoir sur l'oralité, le Mvet découle d'abord du verbe, 
cette parole créatrice qui bien que génitrice, joue également le rôle de censeur dans une société qui lui reconnaît tous les pouvoirs (Emmanuel Matateyou, 2011). Chez les Ékang, l'essentiel de la littérature est constitué de contes et de récits historiques, de mythes et de légendes qui tracent les généalogies, décryptent les énigmes et décodent les proverbes. Les récits mvet ne sont donc pas totalement désintéressés. Le présent article interroge la fonctionnalité de la fiction portée par la parole dans le Mvet. Dans une approche comparatiste entre documentaire historique et fiction, il cherche à démontrer que la fictionnalisation dans le Mvet, loin de chercher à déconnecter l'Ékang de sa réalité, vise plutôt à l'amener à se l'approprier en vue d'une vie authentique pour le « négro-africain ». L'approche comparatiste consistera aussi en l'analyse du Mvet et du documentaire en tant que systèmes (ou sous-parties du système) selon un angle d'approche communicationnel. Toutefois, conscient que la mise en parallèle de ces différents systèmes ne peut suffire à la réalisation de notre étude, pour que cette dernière soit menée à son terme et que son résultat soit crédible, nous allons « repérer leurs différences et trouver une grammaire commune qui permette soit une remise en compatibilité (c'est-àdire une harmonisation), soit une véritable fusion (une hybridation) $\gg$. Ainsi, la méthode comparative convoquée dans cette recherche aura une fonction d'intégration, par un travail de synthèse regroupant des éléments du réel et de la fiction dans le Mvetet pourra permettre, dans certains cas l'hybridation, c'est-à-dire, la «mixtion de règles d'origine diverses ». Il est question dans un premier temps de présenter le Mvet comme une littérature orale qui se sert de la fiction pour traiter du vrai, de la réalité et de l'histoire du peuple ékang. Deuxièmement, nous présentons le Mvet et le documentaire face à leur auditoire. Il est question ici d'établir le pacte, ou du moins le lien qui existe entre le public et ces formes de communications. Enfin nous analysons la fonctionnalité de la fiction dans le Mvet. Il s'agira de démontrer que le joueur de mvet, de manière plus évidente que l'historien, chevauche à la fois les territoires de la réalité et de la fiction, de la science et de l'art, du divertissement et de la transmission du savoir. Le choix du Mvet, en tant que genre épique (Joseph Dong Aroga, 2011), philosophie (Angèle Christine Ondo, 2014) et instrument de musique doté d'une valeur anthropomorphique (Bingono Bingono), apparaît comme le baromètre idéal de la société ékang. Son spectacle nous paraît donc être en la circonstance un territoire privilégié d'observation et d'analyse. 


\section{Mvet et oralité}

Les chercheurs comme Canu Gaston, Eno Bélinga, Calame Griaule, Emmanuel Matateuou etc., ont donné des définitions du paradigme de littérature orale. Dans cette étude, bien qu'y souscrivant, nous précisons avec Kam Sié que cette littérature fait référence à « tout ce qui a été dit, généralement de façon esthétique, conservé et transmis verbalement par un peuple et qui touche la société entière dans tous ses aspects $\gg$. En tant que littérature orale, le Mvet est donc le dépositaire de la connaissance (oyem), de la philosophie et de la sagesse $(\mathrm{mfeg})$ pour le peuple ékang. Il est essentiellement basé sur la parole (ébùk).

\section{Le Mvet, une littérature à part entière}

Le mot littérature désigne à la fois, pour Barthes: «l'art de produire les œuvres d'esprit; l'ensemble des productions littéraires d'une nation, d'une époque; et la connaissance des règles qui doivent diriger ces productions, l'étude des matières et des œuvres littéraires. » Pour Sartre par contre, la littérature est tout simplement, comme il l'a démontré, un moyen de communication. Il s'agit maintenant de savoir ce que l'on veut communiquer, c'est ce que résume la question posée en tête du chapitre « Pourquoi écrire? ». Le Mvet est donc une littérature parce qu'il est un moyen de communication qui dresse une feuille de route du sens de la vie telle qu'elle est et telle qu'elle devrait être vécue par la société ékang dans son époque et dans son milieu à travers le récit, la poésie, la chanson, la danse, les mythes, les métaphores et les rituels religieux. Il englobe les connaissances traditionnelles, les croyances et les valeurs concernant l'environnement et la nature de la société elle-même. Le Mvet apparaît en réponse à l'impulsion esthétique universelle de fournir des récits qui expliquent la nature de la vie et décrient les réponses humaines aux défis qui jalonnent leur existence. Cette littérature décrit comment vivre une vie morale et explique la nature de la relation avec la divinité. Elle conserve ainsi les connaissances de la société à transmettre aux générations suivantes. Le récit mvet contient l'histoire de la société, fournit un guide pour les comportements en communauté (Dénis Bidjo, 2014) et est le censeur du savoir-vivre pour l'Ékang.

Le Mvet est aussi le dépositaire de l'expression artistique dans la société ékang. La richesse et la pertinence de ses épopées résonnent à travers les frontières culturelles des peuples de la forêt équatoriale africaine. Cependant, s'il est établi que le Mvet est une littérature parce qu'il est une réponse à l'instinct humain universel pour trouver l'équilibre, l'harmonie et la beauté dans 
le monde (Edou Alain), il peut donc être à juste titre qualifié de littérature engagée, tant il explique les causes de la souffrance humaine, les justifie et surtout suggère des moyens de médiation et de guérison de cette souffrance (Dénis Bidjo, 2014).

\section{Le Mvet, une littérature engagée}

Une littérature est considérée comme engagée quand « elle présente certaines opinions ou prises de position de son auteur sur un sujet donné. Par le biais de son texte, un écrivain peut critiquer certains aspects de la société, dénoncer une situation qui le dérange ou encore défendre une cause qui lui tient à cœur ». Une personne engagée est de ce fait une personnalité liée à la culture et aux arts qui participent activement à la vie de la cité en exprimant ses choix idéologiques. Il soutient ainsi solidairement des causes qui assurent la promotion des valeurs d'une société ou d'un groupe.

Le Mvet n'échappe pas à cet engagement. Bien au contraire, il cherche à répondre au besoin de croyance religieuse et d'accomplissement spirituel nécessaire à l'existence humaine. Ce monde humain universel, peuplé d'êtres spirituels et de leurs personnalités, est révélé à travers des histoires, des prières et des textes rituels. Le Mvet raconte le travail des dieux, explique comment le monde et l'existence humaine sont nés et révèle la nature de la fragilité humaine (TsiraNdongNdoutoume, 1983). Par ses épopées et les mésaventures de ses héros, il sert à communiquer des idées, des émotions et des croyances. Le Mvet traite de l'aventure humaine et de ses réalisations contre vents et marées. À travers les chants, les rituels et les cérémonies rythmés par cet instrument à cordes composé de plusieurs calebasses greffées à une tige de bambou raphia (Oyeng), les éléments écologiques essentiels à la survie de la société sont décrits et leurs fonctions exaltées. Le Mvet comme littérature orale est aussi une forme de divertissement qui favorise le sentiment de solidarité envers les personnes qui ont vécu des expériences douloureuses de la vie (Lluís Mallart Guimera, 2009). En résumé, cette littérature orale englobe de nombreux genres d'expression linguistique et remplit par l'utilisation de la voix, de nombreuses fonctions différentes dans la société, d'où sa comparaison avec le film documentaire. La comparabilité de ce point de vue ne se détermine pas, car aucun système n'est à tout point identique à un autre. Plus précisément, bien que les conditions de communication qui existent dans le Mvet et le documentaire ne sont pas identiques, leur organisation et leurs relations au public révèlent néanmoins des similitudes. 


\section{Documentaire et Mvet: deux outils de communication au service de l'éducation}

Le documentaire comme le Mvet ont d'abord comme point commun une fonction éducative (Bingono Bingono, 2017). Un film documentaire, comme une épopée, peut s'appuyer sur une narration vocale pour décrire ce qui se passe en images réelles ou virtuelles. Pour agir sur leur auditoire, les films documentaires se fondent sur la vie réelle et incluent des séquences d'événements racontées par une voix «in $»$ ou «out $»$, c'est-à-dire faisant partie ou non de la diégèse. C'est un avantage par rapport aux autres modes de divertissement créés pour le grand public: le spectateur ne voit pas ce qui lui est raconté dans le vide. En général, le public du Mvet comme celui du documentaire connaît déjà un aspect du sujet et l'instructeur qui est la parole du mbomomvet ou la voix-off du film peut à partir de ce moment épiloguer, expliquer et renseigner sur la complexité du problème auquel l'histoire se rattache. Il a donc toujours existé de manière consciente un pacte, voire un partenariat tacite entre l'aède mbomomvet et son auditoire, tout comme entre le cinéaste documentariste, représenté lors de la réalisation du film par la voixoff, et son public. En se rendant à un spectacle de Mvet, le spectateur sait que l'histoire qui lui sera racontée n'est pas vraie, mais il y adhère et choisit de se laisser transporter comme le cinéphile d'une fiction, dans le monde imaginaire et imagé de l'auteur. Dans les deux cas, malgré le fait que l'auditoire est virtuellement captif, le documentaire et le Mvet doivent toujours s'impliquer et s'inspirer du vécu réel pour divertir davantage. L'objectif central pour ces genres est de révéler au public ce qu'il croit savoir alors qu'il ne le sait pas. Comme l'acteur à la recherche d'un personnage, le Mvet est à la recherche de son public. Un public dont la participation au conte par le chant, la danse, les battements de mains et les cris d'approbation participent de la diégèse et de l'oralitureékang exaltant dans un passé imaginaire les hauts faits du peuple éponyme, d'où son assimilation au documentaire historique.

\section{Le Mvet, le documentaire historique ékang}

Le documentaire historique est un genre cinématographique qui relate l'Histoire à partir d'éléments ayant valeur de preuves : les documents. Il peut donc se rapporter au Mvet qui lui, est la narration de l'histoire du peuple ékang à partir du document sonore qu'est la voix du conteur. Pour mieux apprécier cette comparaison qui peut sembler triviale, il faut juste se référer à la place et à l'autorité que représente le mbomomvet et surtout, comme le souligne Angèle Christine Ondo (2010), au rôle de la parole dans le Mvet. 
[...], dans le discours des héros et des médiateurs de la transcendance, la parole se subdivise en « parole ordinaire » et en « parole force ». Cela signifie que les « paroles forces » ont le pouvoir de transformer la matière, notamment dans les initiations.

Par ailleurs, la bouche constitue la principale voie par laquelle sortent des armes secrètes des guerriers du Mvet. Ces attributs ne s'utilisent que dans les temps forts représentés par les combats et l'initiation. La parole dans le Mvet est donc une parole-force, une parole agissante et respectée. Elle est une arme pour l'Ékang. Ce respect de la parole est à juste titre transposé au mbomomvet dont la parole devient alors une parole-témoin de l'histoire qu'il raconte, une parole-document. On peut donc assimiler la parole du conteur à la preuve dont l'historien a besoin pour écrire l'histoire. L'histoire est une représentation du passé qui reflète les intérêts et les préjugés des praticiens de la discipline. Comme le souligne Richard White dans un essai, l'un des principes fondamentaux de l'histoire comme discipline, c'est que le passé est différent de notre propre temps, d'où la nécessité de s'appuyer sur les documents. Seulement, les outils de persuasion du mbomomvet ne reposent pas sur la documentation écrite pour soutenir son argumentaire, mais plutôt sur sa capacité d'examiner les situations en profondeur et de peser la validité des diverses interprétations. Cette approche exige non seulement un engagement intellectuel de la part du conteur mais aussi de celui du spectateur. Le présupposé accès aléatoire à l'histoire par l'interprétation limitée du spectateur du documentaire ne trouve pas un écho favorable dans le Mvet. Ici, le sentiment de la possibilité d'une interprétation erronée du public n'est qu'une impression. Certes la compréhension du message n'est pas à la portée du premier venu, c'est-à-dire du spectateur non averti, mais la lecture du Mvet est univoque : les peuples Beti- Bulu- Fang et tous les clans qui leur sont apparentés ayant tous le même référant. Ses références au présent, au futur et à l'au-delà facilitent cette conception et cette compréhension du passé. Les spectateurs peuvent recevoir l'épopée et l'«ingurgiter » à leur propre rythme; il s'agit dans ce cas de figure de les amener à arrêter de réfléchir sur les idées et les modes de pensée qui leur sont étrangers, et à se tourner vers le mbomomvet, telle la voix-off d'un documentaire, pour suivre les arguments de son explication.

L'épopée mvet est également une construction reflétant une certaine forme. Les conteurs cherchent à toucher émotionnellement les spectateurs par la création de l'histoire et des personnages. Une façon de créer ce lien est de faire en sorte que les téléspectateurs pensent que les personnages du conte sont des gens comme eux. Par conséquent, de nombreuses épopées 
mvet soulignent les points communs du comportement des héros mortels et immortels plutôt que leurs différences. Ces protagonistes d'un bord comme de l'autre traversent les frontières temporelles et culturelles, défient les lois de la pesanteur et sont d'un orgueil démesuré.

Cette convention pour établir la familiarité avec son auditoire est due en grande partie au mode de présentation. L'épopée est un récit historique basé sur des événements et des faits attribués à un individu de naissance noble. C'est le cas de l'épopée de Ndzana Ngazogo et celle d'Ateba Ebe chez les Éton, ou celle de Charles Atangana chez les Éwondo ${ }^{1}$. Ici, le spectateur n'a aucun contrôle du flux de la transmission de l'information. L'information doit donc être facilement comprise. Elle se fait généralement par une narration linéaire, mais avec une argumentation complexe truffée de proverbes, d'idiotismes et de séjours dans le bekon (monde des morts). Ces moyens de persuasion sont principalement mis en scène par la juxtaposition des mots, des tournures langagières imagées et des chants de l'aède que reprend en chœur l'assistance.

\section{De la morphologie du conte et du documentaire: les points de similitudes}

Le mbomomvet s'efforce de trouver un équilibre entre l'envie du public, de découvrir le passé comme terrain familier et la prédilection du poète pour souligner son étrangeté. Pour ce faire, l'épopée est structurée chronologiquement et divisée en deux parties distinctes : la narration proprement dite de l'histoire et le chant rythmé par l'instrument mvet. Comme le film documentaire, l'épopée explore aussi la société contemporaine par ses allusions et ses références. Elle présente les enjeux centraux de la survie de la communauté tels que la croyance en la divinité suprême Éyo, la préservation des traditions spécifiques aux Ékang, la transmission de la connaissance à la jeune génération et la participation à la résolution des problèmes de la communauté. De nos jours, des problèmes sociaux plus actuels tels que la classe sociale, le rôle de l'éducation, de l'alphabétisation et du genre sont également abordés.

Le Mvet est un «spectacle-document» historique qui présente sous forme de récit la prestance de la caste mythique ékang. L'épopée commence par un chant interactif qui peut être considéré comme chant de ralliement. Le mbomomvet et le public approbateur clament avec magnificence l'arrivée des descendants Ékang en ces termes ${ }^{2}$ : 
Mbomomvet
ékangbissobissoélangélangééé ! (les Ekang sont de passage !)

Public

ééééééée (cris d’approbation)

\section{Mbomomvet}

ma dzonékangbissoélangélangéée! (je dis bien que les Ekang sont de passage!)

\section{Public}

ééééééé

\section{Mbomomvet}

bi man yasòò a mot ékangéé! (nous sommes arrivés, frères Ekang!)

\section{Public}

ééééééé

\section{Mbomomvet}

melò me bààà? (avec combien d'oreilles m'écoutez-vous?) $)^{3}$

\section{Public}

mebàààò (avec deux d'oreilles) ${ }^{4}$

\section{Mbomomvet}

ékangmboloo! (noble peuple de guerriers Ékang, je te salue!)

\section{Public}

essangom (nous acceptons le salut)

\section{Mbomomvet}

madzonékangmbolo! (je dis bien, noble peuple de guerriers Ékang, je tes salue!)

\section{Public}

essangom (nous acceptons le salut)

\section{Mbomomvet}

ékangmboloo! (noble peuple de guerriers Ékang, je te salue !)

\section{Public}

essangom (nous acceptons le salut) 


\section{Mbomomvet Ékang!) \\ Public \\ Ayaaaaaaaaaaaaaaaaa}

essangomaababetoaa! (voici rassemblés le noble peuple de guerriers

Ce prélude, tel un générique de début de film, a pour but non seulement d'annoncer le début de l'épopée mais aussi de solliciter l'attention de l'auditoire qui doit se mettre dans les conditions les meilleures pour recevoir la sagesse du conteur (Dénis Bidjo, 2017). En effet, il est fréquent que certains joueurs de mvet aient des interdits. C'est le cas du grand maître Akué de la contrée Ntumu au sud du Cameroun, qui arrêtait systématiquement sa prestation lorsqu'il constatait qu'un membre de l'auditoire consommait du concombre sous quelques formes (OssimaMenyé, 2017). Ce chant résonne donc comme une annonce ou mieux, un avertissement au public à se débarrasser de tout élément pouvant contrarier le conteur. Cela fait, le barde peut alors prendre possession de son public et le balader dans son univers où les faits d'armes des Ékang sont retracés avec emphase dans un réel métaphorique, un réel savamment fictionnalisé.

\section{La fonctionnalité de la fiction dans le mvet}

Le génie créateur du conteur utilise la fiction. Il raconte des histoires auxquelles il prétend avoir assisté comme témoin. Pour certains spectateurs, c'est une expérience enrichissante ; pourtant, l'exubérance et l'imagination débordante ne sont pas toujours compatibles avec les obligations de l'historien. Le Mvet est un mélange de faits historiques et de fiction utilisés sous diverses formes, où récits et sagas participent de la construction de l'imaginaire ékang. L'épopée est une tentative de satisfaction du désir des auditeurs à maîtriser les enjeux des combats qui opposent les mortels aux immortels. C'est une séance d'apprentissage et de divertissement en même temps, basée sur la fiction. L'auditeur semble éprouver un besoin d'authenticité malgré la « dénégation $^{6} \gg$, car il sait que ce qui lui est raconté n'est qu'une œuvre de fiction.

Contrairement à l'historien, le mbomomvet ne fonctionne pas avec le recul que devrait lui imposer le maniement des événements passés. Il vit dans la conscience de ses personnages pour qui le futur est un vide. Or, l'historien fait les deux - d'abord expliquer le monde tel qu'il apparaissait aux protagonistes de l'époque, puis l'analyser avec recul. Bien souvent, le conteur prête ses pensées à une figure historique ayant véritablement existé. On pourrait 
même dire que le conteur s'aventure dans un «terrain dangereux » lorsqu'il modifie de vrais événements. Dans ses récits, il va jusqu’à faire dire aux personnages ayant véritablement existé, des paroles dont la véracité n'est pas prouvée. C'est le cas du mbomomvet Andzeng Etaba qui dans l'épopée de Ndzana Ngazogo, fait le récit de la conversation du personnage éponyme avec la déesse que celui-ci a rencontré dans l'au-delà. Cependant à bien y voir, le mbomomvet est scientifiquement honnête. Bien qu'en utilisant la fiction, il donne des indices à son auditoire sur l'authenticité ou non de l'histoire déclamée. Par l'anastylose, les archéologues, restaurateurs de peintures et de poteries suivent un code de conduite dans leur travail pour distinguer le matériel authentique et original de ce qu'ils ajoutent plus tard. Le mbomomvet en fait de même. Lors de son récit, il dit à sa manière au spectateur du conte ce qui est authentique et ce qui est inventé. Et quand bien même certains conteurs ne feraient pas cette distinction (surtout qu'à l'oral il est impossible d'utiliser les guillemets, l'italique ou le gras pour distinguer le vrai de la fiction) ne changent-ils pas légèrement les noms de véritables protagonistes, pour souligner que l'histoire déclamée est partiellement vraie ou n'est pas du tout la réalité ? La romancière Linda Grant a d'ailleurs fait valoir, concernant le roman, que cela donne à l'écrivain une plus grande liberté d'invention. Pour elle, « garder de vrais noms entrave l'imagination, peut-être plus que ça ne la réalise $^{7} \gg$. Dans les épopées Mvet, les personnages les plus admirés et les plus intéressants sont ceux inventés de toutes pièces, et non les personnages historiques, c'est-à-dire ayant effectivement existé. Dans son article « L’espace corporel intérieur dans le mvet $\gg^{8}$, Angèle Christine Ondo a dressé la liste des personnages suivants :

\section{Mvet 3 : Le Mvet : L'Homme, la mort et l'immortalité9}

Les héros mortels

- Obame Andome Ella (Pays Essighlessi)

- Andome Ella Mezang (le père du héros)

Les héros immortels des quatre œuvres

$\begin{array}{ll}\text { - Akoma Mba } & \text { (chef du pouvoir politique) } \\ \text { - Angoung Bere } & \text { (chef du pouvoir spirituel) } \\ \text { - Engwang Ondo } & \text { (La triade) } \\ \text { - Angone Endong } & \text { (La triade) } \\ \text { - Ntoutoume Mfoule } & \text { (La triade) }\end{array}$




- Obiang Medza
- Medza Me Mfoule
- Ondo Biyang
- Nguéma Nsing Bere
- Ze Medang
- Efoua Medang
- Ayangoum

- Obiang Medza

- Medza Me Mfoule

- Ondo Biyang

- Nguéma Nsing Bere

- Ze Medang

- Ayangoum
(Les jeunes guerriers)

(Les jeunes guerriers)

(Les jeunes guerriers)

(Les jeunes guerriers)

(Les jeunes guerriers)

(Les jeunes guerriers)

(Le grand magicien Ayakoma des autres peuples)

Il en est de même pour les personnages les plus mémorables dans la littérature et au cinéma; ils sont toujours issus de l'imagination d'un auteur. Il pourrait sembler logique que la réalité et le vrai avec ce qu'ils supposent comme rigueur dans la démarche, soient en fait des instruments plus persuasifs du changement social que la fiction; mais ce n'est pas forcément le cas. Tandis que la fiction ajoute à la connaissance du lecteur, elle transforme le paysage interne de ce dernier dans un monde bien réel, le monde actuel. Ceci nous amène à présent à questionner le rôle du Mvet dans la société contemporaine. Autrement dit, en quoi le récit du Mvet essentiellement basé sur des faits fictionnels et historiques, donc relevant du passé, permet-il à l'Ékang de s'approprier le monde actuel.

\section{Mvet et contemporanéité}

Aujourd'hui encore, chaque épopée met en avant un argument logique et une théorie de la cause et de l'effet par des phrases séquencées. Le récit est entrecoupé des chants du conteur et des réponses du public. Si le costume a évolué, la technique est restée la même. Le conteur et l'auditeur savent tous les deux que si le premier ne fournit pas la logique du récit, le second le fera. Mais la logique des événements et des gens tels qu'ils existent dans le monde du Mvet ne va pas de soi. Les mbomomvet, donc narrateurs de fiction et les documentaristes, supposés ne narrer que la réalité, ont des manières différentes de rassembler leurs systèmes logiques. Pour les documentaristes, l'histoire est basée sur des faits connus, et généralement acceptés pour être vrais. Les faits du documentaire sont comme des trouvailles scientifiques - ils doivent nous apparaître comme tangibles et réels, donc probables, plausibles, attestés, mais aussi nouveaux et révélateurs. La promesse scientifique du documentaire vient apporter un gage de précision, et donc de conformité au réel. Le côté scientifique du documentaire concerne ce que ces faits ou objets pourraient signifier, comment ils pourraient être reliés à l'histoire pour laquelle ils constituent des preuves. 
Dans le conte mvet par contre, les auditeurs veulent savoir non seulement ce qui s'est passé, à quoi le monde qui leur est présenté dans la fiction ressemblait, mais aussi à quoi il serait l'équivalent aujourd'hui. L'auditoire veut savoir mais aussi expérimenter, et ainsi il suspend volontairement l'incrédulité à la fiction. Ce que les spectateurs du Mvet obtiennent de cette source n'est pas seulement le plaisir, mais l'éducation, l'exercice de l'imagination, le questionnement intérieur... Le conteur a aussi une théorie sur le passé. Pour lui, le passé et le présent sont similaires, bien que les gens changent ou restent les mêmes. Comme l'historien ou le documentariste, le mbomomvet pour construire son récit, fait beaucoup de recherches. Mais il sait que la réussite de sa méthode ne sera pas due à l'exactitude de son récit, mais plutôt à sa capacité de saisir la nature humaine dans l'immédiateté. Il est alors question d'adapter l'histoire au contexte qui fait l'actualité et à son époque. Pour mieux appréhender cette fictionnalisation de la réalité, nous nous référons aux résumés des épopées contenues dans « La naissance du mvet », publié par le blog « mone fang ye gong é monenzame a vine ayen »:

Aloum Ndong Minko et Ngâne sont deux autres célèbres récits connus du monde Fang. [...] AloumNdongMinko est l'homme puissant du Nord qui ne construisait qu'avec la peau des hommes. Son entreprise - ôter la vie à quarante humains - fut écourtée lorsqu'il prit la peau du beau-père de deux immortels, car ces derniers lui livrent une guerre sans merci. [...] Abiè est un récit qui met à jour les querelles intestines entre les immortels. En effet, un religieux d'Engong, inquiet de la montée du poker dans le village, se décida de l'interdire en passant par l'ultime jeu dont le gagnant resterait le seul joueur à Engong. La querelle intervient au moment où l'initiateur du jeu veut passer outre le règlement $d u$ jeu, pourtant établi par lui-même. Biang Duma ou le fétiche de la célébrité, est [un] autre récit d'Akuè Obiang. Ce récit met en évidence un immortel, Effoua Medang, qui, frustré d'être moins célèbre que son cadet, alla consulter le féticheur du village, Angoung Bere, qui lui demande en contrepartie de tuer sept belles-mères d'Engong. Effoua Medang respecte ces consignes en tuant six bellesmères. Mais le problème se pose lorsqu'il tue la septième, cette dernière étant celle d'Akoma Mba, chef suprême d'Engong. [...] le plus célèbre de tous est le dernier, L'homme, la mort et l'immortalité, publié chez L'Harmattan. Dans ce récit, Obame Andome Ella se décide d'aller demander à son oncle Akoma Mba les raisons du meurtre de son père. Mais c'est une mission quasi impossible, car Angone Zok Endong Oyono a construit un pont de boas sur le fleuve Dzame Anene, frontière naturelle entre le pays des mortels et le pays des immortels ${ }^{10}$.

C'est en cette intrusion dans la fiction pour dire la réalité que le Mvet est supérieur aux autres genres. Non seulement parce qu'il reflète par la fiction 
un processus intrinsèquement plus créatif, mais parce quau mieux, il est capable par l'actualisation de ses épopées, désensibiliser son auditoire avec une richesse, une complexité et une profondeur qu'aucun autre genre (écrit ou autre) ne peut atteindre. Comme le conte africain en général, «c'est un moyen d'éducation intellectuelle à partir duquel se forme et se prépare l'esprit de l'enfant et de l'adolescent pour leur participation efficace et honnête à la vie de leur communauté ${ }^{11} \gg$.

D'un autre côté, par excès de zèle, le conteur utilise sa filiation comme adjuvant, dans une forme narrative où il s'abrite lui-même derrière un personnage de son récit pour toucher son auditoire. Pour les mbomomvet comme Dénis Bidjo, c'est un excellent moyen de démontrer la pureté de sa descendance ${ }^{12}$. C'est ainsi qu'il est fréquent que les épopées commencent par la déclinaison de la généalogie du conteur. Et pour que cette attitude narrative soit conduite jusqu'à son terme, le mbomomvet laisse entendre que son texte est une confession, il appelle ainsi à une écoute en partie référentielle. La personnification dans le mvet n'est donc qu'à demi-fictive, son contenu et l'effet qu'elle recherche sont aussi autobiographiques. À l'opposé, la fictionnalisation de soi par le mbomomvet consiste à inventer des aventures qu'il s'attribuera. Et pour que cette fictionnalisation de soi soit totale, le conteur donne à cette invention une valeur figurale ou métaphorique. Il encourage ainsi une «lecture référentielle ${ }^{13} \gg$ qui déchiffrerait dans son texte des confidences indirectes. Mais il y a des limites dans la mesure où nous pouvons compter sur des films pour transmettre la vérité historique. Comme la plupart des films historiques populaires, le Mvet bien qu'il ne soit pas à proprement parler un documentaire, mais une représentation dramatique, raconte une histoire intéressante, dépeint des personnages fascinants aux costumes qui semblent nous ramener à l'époque du récit. Or, dans quelle mesure pouvons-nous faire confiance au Mvet (ou à toute autre dramatisation de l'histoire) comme source de faits historiques? Le Mvet peut présenter des événements historiques, de façon vive et édifiante peut-être, mais il n'a pas de place pour témoigner en faveur de la vérité des faits présentés. En conséquence, en écoutant simplement le conteur, nous n'avons aucun moyen de savoir dans quelle mesure « le discours » est précis, exact.

Il en est de même pour le documentaire. On pourrait penser que les questions sur l'exactitude du film sont résolues une fois que nous apprenons que, contrairement à de nombreux films historiques, il utilise sérieusement les sources historiques et les interprétations des historiens. Dans les documentaires de reconstitution (docufiction) on annonce même souvent en générique que le film est basé sur une histoire bien réelle, et d'éminents his- 
toriens sont répertoriés comme consultants pour attester de leur véracité. Pourtant, le film, tout comme le mvet, ne peut pas fournir toutes les sources et les arguments qui pourraient soutenir les innombrables décisions de ses responsables techniques et artistiques. De plus, étant donné qu'il s'agit d'une dramatisation de l'histoire bien que ce soit à travers des documents supposés vrais, les cinéastes documentaristes - même les plus rigoureux et fidèles ont toujours des partis pris. Que ce soit dans les angles de prise de vue ou les choix de l'angle d'attaque, ils exercent chacun en fonction de son degré d'implication, une influence sur le réel présenté. Le mbomomvet et le documentariste ont donc aussi ce point en commun, ils exercent une influence sur l'histoire qu'ils présentent, d'où a question sur l'objectivité et l'exactitude des faits historiques dans une œuvre artistique émanant de la perception (donc forcément limitée) de son auteur.

\section{Conclusion}

Cette étude examine la relation entre le Mvet, récit épique du peuple ékang et le cinéma documentaire historique dans une perspective comparatiste. Il s'agissait donc d'une approche interdisciplinaire qui avait pour but de positionner le Mvet comme un outil, une technique, une technologie, voire, une discipline au service de l'histoire du peuple des « seigneurs de la forêt », les Ékang. Pour ce faire, nous avons analysé la fonction de la fictionnalisation du réel dans les épopées mvet. En somme, on peut dire que la division entre fiction et réalité dans le Mvet est donc intrinsèquement fausse selon la théorie du multivers, en ce sens que toute fiction est vraie dans un univers ou dans un autre. Alors, quand le mbomomvet psalmodie, il conte une réalité qui appartient à un univers particulier. Mais il y a une autre raison pour laquelle la division est fausse, ou du moins pourquoi elle crée des idées fausses. Les choses classées comme réelles, donc supposément vraies, peuvent être inauthentiques alors que la fiction peut contenir plus de vérité. Le but du Mvet, même en s'appuyant sur la fictionnalisation du récit et l'imagination de l'aède, est la poursuite de la vérité. L'expression «prêcher le faux pour avoir le vrai » trouve tout son sens ici. La fiction dans le Mvet a pour but d'amener l'Ékang à s'approprier le monde réel, à le façonner et à l'impacter. Le Mvet par la fictionnalisation de ses récits prêche les valeurs vers lesquelles les Ekang doivent tendre. Il s'agit de la conscience de l'existence du Dieu suprême Éyo, le respect des anciens et de la parenté, le dévouement à la communauté, bref l'amour du prochain. Le Mvet est donc la philosophie ékang. Il accompagne et guide l'Ékang dans sa quête de l'éternité qui est dans ce monde, l'accès au statut 
d'ancêtre après la mort. En effet, comme dans les combats entre les mortels et les immortels, le but de la vie pour l'Ékang est la recherche du secret de l'immortalité, de l'éternité c'est-à-dire de la vie après la mort. Par le respect des préceptes et valeurs qui réglementent la vie en communauté et fondent la philosophie ékang, l'homme après sa mort est digne d'entrer dans le monde des ancêtres. Les épopées mvet posent la question de l'être, celle de la nature et du devenir de l'homme après son pèlerinage terrestre. Par l'acceptation de sa mort, après une vie passée au service de la communauté et du bien, le Mvet invite l'Ekang à l'élévation, à transcender l'angoisse que peuvent générer les problèmes existentiels. L'anthropologue Bingono Bingono dira d'ailleurs que le mot «mvet » vient du verbe abet qui signifie s'élever. L'expression Ma bommvet (je joue du mvet) signifierait selon lui dans la langue ékang « je m'implique dans une activité d'émergence, de progrès ${ }^{14} \gg$. De nos jours, en décrivant les travers des hommes, en dénonçant les situations d'injustice, en ouvrant des débats philosophiques, politiques et sociaux selon les cas, le Mvet est le thermomètre de la société ékang en prise aux nouveaux défis que lui impose la mondialisation. Le Mvet se transforme, se réinvente chaque jour et se « re-crée » afin de répondre aux besoins d'un environnement et d'un monde en perpétuelle mutation. Si de nos jours les séances publiques de Mvet se font de plus en plus rares, et que de grands mbomomvet ont tiré leur révérence, le Mvet lui reste bien vivant. Ses récits d'une étrange originalité et ces combats entre mortels et immortels continuent d'agrémenter les épisodes de la vie des Ékang avec une fictionnalisation d'un réel déconcertant. Il reste d'actualité et participe de l'initiation contemporaine à la vie... et à la mort. Surtout que, comme le reconnait Cécile Filliatre, «... tout conte peut être initiatique dans la mesure où il nous apprend toujours quelque chose ${ }^{15} \gg$. 


\section{Sources orales}

\begin{tabular}{|c|c|c|c|}
\hline $\begin{array}{l}\text { Noms } \\
\text { et prénoms }\end{array}$ & Âges & Qualités & $\begin{array}{c}\text { Date de } \\
\text { l'entretien }\end{array}$ \\
\hline Bingono Bingono & 68 ans & $\begin{array}{l}\text { Patriarche Beti, } \\
\text { anthropologue }\end{array}$ & 18 mai 2017 \\
\hline Andzeng Etaba Pantaléon & 65 ans & Patriarche Éton, mbomomvet & 10 juillet 2017 \\
\hline Edou Alain alias Vinangor & 52 ans & Mbomomvet Fang & 23 août 2017 \\
\hline Ossima Menye & 75 ans & Patriarche Ntumu & 20 août 2017 \\
\hline Bidjo Dénis Blaise & 66 ans & $\begin{array}{l}\text { Mbomomvet, patriarche } \\
\text { Bulu, Département de } \\
\text { communication africaine, } \\
\text { ESSTIC-Cameroun }\end{array}$ & 20 juin 2017 \\
\hline
\end{tabular}

\section{Notes}

1. Les Eton et les Ewondo sont des sous-groupes du peuple ékang qui vit au Cameroun.

2. Cette traduction en langue française s’inspire de celle de Gervais Mendo Zé dans La forêt illuminée, éd. Société Nouvelle Firmin-Didot, 1988.

3. Comme pour dire "prêtez-moi votre attention ».

4. Comme pour dire « nous sommes tout ouïe».

5. Onomatopée qui marque l'approbation unanime de la foule.

6. Anne Ubersfeld, Lire le théâtre t. I, Paris, Éditions sociales, 1977.

7. Linda Grant, The People on the Street, a writer's view of Israel, Londres, Virago Press, 2006.

8. Angèle Christine Ondo, op. cit.

9. Tirée du titre de l'œuvre de TsiraNdongNdoutoume, Le Mvet, l'Homme la Mort et l'Immortalité, l'Harmattan, 1993. 
Lecture documentarisante et fictionnalisation du réel dans le spectacle Mvet du peuple ékang

10. http://maximehenry2.skyrock.com/1626924816-LA-NAISSANCE-DU-MVETT. html, [consulté le 31 octobre 2017 à 7 h 20].

11. Cécile Filliatre, «Le conte africain, une leçon de vie. Oralité -Écriture -Théâtre Citoyenneté », Dossier PAC, Académie de Créteil, 2006.

12. Entretien avec le conteur Dénis Bidjo, le 20 juin 2017.

13. Vincent Colonna, L'autofiction, essai sur la fictionalisation de soi en littérature, Linguistique, École des Hautes Études en Sciences Sociales (EHESS), 1989. 